\title{
Exploring the Protein G Helix Free Energy Surface by Solute Tempering Metadynamics
}

\author{
Carlo Camilloni, ${ }^{1,2}$ Davide Provasi, ${ }^{1,2}$ Guido Tiana, ${ }^{1,2, * *}$ and Ricardo A. Broglia ${ }^{1,2,3}$ \\ ${ }^{1}$ Department of Physics, University of Milano, via Celoria 16, 20133 Milan, Italy \\ ${ }^{2} I N F N$, Milan Section, Milan, Italy \\ ${ }^{3}$ The Niels Bohr Institute, University of Copenhagen, \\ Blegdamsvej 17, DK-2100 Copenhagen, Denmark
}

(Dated: October 30, 2018)

\begin{abstract}
The free-energy landscape of the $\alpha$-helix of protein $\mathrm{G}$ is studied by means of metadynamics coupled with a solute tempering algorithm. Metadynamics allows to overcome large energy barriers, whereas solute tempering improves the sampling with an affordable computational effort. From the sampled free-energy surface we are able to reproduce a number of experimental observations, such as the fact that the lowest minimum corresponds to a globular conformation displaying some degree of $\beta$-structure, that the helical state is metastable and involves only $65 \%$ of the chain. The calculations also show that the system populates consistently a $\pi$-helix state and that the hydrophobic staple motif is present only in the free-energy minimum associated with the helices, and contributes to their stabilization. The use of metadynamics coupled with solute tempering results then particularly suitable to provide the thermodynamics of a short peptide, and its computational efficiency is promising to deal with larger proteins.
\end{abstract}

Keywords: Free-energy Surface, Metadynamics, Solute Tempering, protein G, $\alpha$-helix, $\pi$-helix.

*Corresponding author: guido.tiana@mi.infn.it; address:Department of Physics, via Celoria, 16, 20133 Milano, Italy; phone:+39-02-50317221

${ }^{\dagger}$ Short title: Free energy of protein-G helix 


\section{INTRODUCTION}

The B1 domain of streptococcal protein G (Protein G thereafter) is the immunoglobulin binding domain of the protein and comprises 56 residues located at its N-terminus. The folding of this small protein has been studied thoroughly, and accurate structural and thermodynamic characterizations are available for it. The domain is a stable globular folding unit with no disulfide cross-links, and in its native fold a central $\alpha$-helix is packed against a four-stranded $\beta$-sheet, formed by two anti-symmetrically disposed $\beta$-hairpins [1]. The folding occurs without detectable intermediates. Differential scanning calorimetry [2] and stopped-flow mixing methods [3] show that the protein exhibits a two-state unfolding behavior over a wide pH range, and that the kinetics of folding and unfolding can be fit to a single, first-order rate constant over a wide temperature range.

Interestingly, the three fragments of secondary structure have different stabilities when isolated from the remainder of the protein; in particular, the second $\beta$-hairpin (comprising residues 41 through 56) is the most stable one, while the helix (residue 21 through 40) and

the first $\beta$-hairpin (residues 1 through 20) are unstructured in water [4]. Moreover, the $\alpha$ helix fragment has been found to be stabilized by some non-native hydrophobic interactions with its C- and N-terminus residues. [5]. Finally, replacing the helix wild-type sequence at residues 21 through 40 with the second-hairpin sequence, the same native fold is obtained, suggesting that are the non-local interactions due to the $\beta$-hairpin that determines the fold of protein G [6].

By studying the effects of point mutations in protein G, McCallister, et al. [7] suggest that its transition state is characterized by a largely structured second $\beta$-hairpin forming three stranded $\beta$-sheet with the N-terminal $\beta$-strand of the first hairpin. In the transition state the helix seems only partially formed towards the C-terminus region.

Although such intense experimental work provides interesting information about the folding dynamics of protein $\mathrm{G}$, the extremely complex nature of the process makes it hard to rationalize its details, making the problem well suited for a computational analysis. While it would be computationally easy to make standard unfolding simulations of a 20-residues peptide, we pursue a more ambitious goal: to calculate its equilibrium free-energy landscape. With standard molecular dynamics simulations, this is reached only after a large number of folding and unfolding events, corresponding to many crossing of barriers whose height is 
much larger than $k_{b} T$.

To overcome this limit and explore the thermodynamic properties of such systems within all-atom, explicit solvent models, several different methods have been proposed. Among them are: a) the parallel tempering method [8, 9] that allows the system to diffuse faster along its phase space by stochastically swapping different replicas with different temperature; b) the metadynamics [10,11, 12], where the system is allowed to climb over large free-energy barriers by introducing a non-Markovian potential defined as a function of a set of few collective variables (CV) which disfavor the exploration of region already sampled. These two different approaches were combined recently [13] to get the free energy surface (FES) of protein-G second $\beta$-hairpin.

The application of such method to larger systems, however, is hampered by the need to use a big number of replicas in order to ensure a proper rate of exchange. The acceptance probability of a swap between two replicas is proportional to $\exp (\Delta \beta \Delta E)$, where $\Delta \beta$ is the difference of the inverse of replicas temperatures times the Boltzmann constant, and $\Delta E$ is the replicas' energy difference. The larger the system (and thus the larger $\Delta E$ ), the smaller the difference in temperature between replicas and thus the larger the number of replicas needed for a fast equilibration. This computational limitation prevents the use of parallel tempering for systems larger than $\sim 10$ residues. To overcome this limit Liu, et al. [14], proposed a replica-exchange solute tempering algorithm which reduces the number of replicas needed for an efficient equilibration, thus allowing the study of larger systems.

In this work we combine metadynamics and solute tempering for the first time, and use it to hike the FES of the $\alpha$-helix of the protein G. We show that the combined action of the two approaches constitutes a powerful method to study the thermodynamic properties of large complex systems at the all-atom level with explicit solvent. Furthermore we demonstrate that metadynamics and solute tempering is computationally more affordable than parallel tempering metadynamics.

From the sampled FES we are able to reproduce and to provide an interpretation of several experimental findings. Our calculations correctly show that the helix is not stable in water, that the metastable helical state is shorter than the helix as it is found in the protein. Interestingly also $\pi$-helical structures are found. Furthermore, we observe the presence of partial $\beta$-structures in the unfolded region. Moreover we try to characterize some aspects of the helix formation pathway and non-native interaction that stabilize the metastable helix. 


\section{MATERIALS AND METHODS}

\section{A. Theoretical framework}

In parallel tempering a set of replicas evolving at increasing temperatures $T_{m}=\left(k_{B} \beta_{m}\right)^{-1}$ are swapped within the replica exchange algorithm, a procedure which improves the correct sampling of the canonical ensemble for all the replicas.

The solute tempering algorithm allows to dramatically reduce the number of replicas needed in a parallel tempering run, by allowing the potential energy to scale with temperature in such a way that the molecule of interest appears to get hotter, but water stays cold as one climbs the replica ladder. Liu, et al. [14] devise a rigorous transformation in which acceptance probability for replica exchange, scales only with the number of degrees of freedom of the biomolecule but not with the number of water molecules.

The total interaction energy is written as a sum of three contributions: a protein-protein interaction term $E_{p}$, a protein-water interaction $E_{p w}$ and water-water interaction $E_{w}$. The colder replica evolves under the action of the true interaction at the physical temperature $T_{0}=\left(k_{B} \beta_{0}\right)^{-1}$ while the warmer replicas, which evolve at $T_{m}=\left(k_{B} \beta_{m}\right)^{-1}$, have their interaction rescaled as

$$
E_{m}=E_{p}+\frac{\beta_{0}}{\beta_{m}} E_{w}+\sqrt{\frac{\beta_{0}}{\beta_{m}}} E_{p w} .
$$

Since the potential energy surfaces of all the warm replicas are rescaled, only the cold replica sample the correct distribution in the phase space. Following the approach of Bussi, et al. [13], we complement the improvements due to the replica exchange algorithm with those of metadynamics [10, 11, 12].

We consider $N$ replicas of the system. We run them in parallel at increasing temperatures and, with a frequency $\tau_{x}^{-1}$, exchange between two adjacent replicas is attempted. Each replica evolves under the action of the force field and of an adaptive bias potential $V_{m}(\vec{s}, t)$ calculated using the metadynamics algorithm. This potential acts on a set of collective variables $\vec{s}(X)$ defined as a function of the microscopic coordinates $X=\left\{\vec{R}_{1}, \vec{R}_{2}, \ldots\right\}$. With a given frequency $\tau_{G}^{-1}$, the bias potential is updated adding a Gaussian hill, so that the potential is given by

$$
V_{m}(\vec{s}, t)=w_{m} \sum_{t_{k}<t} \exp \left(-\sum_{i} \frac{\left(s_{i}-s_{i}\left(X_{m}\left(t_{k}\right)\right)\right)^{2}}{2 \sigma_{i}^{2}}\right),
$$


where $X_{m}\left(t_{k}\right)$ are the microscopic coordinates of the $m$-th replica at time $t_{k}, w_{m}$ gives the height of the hill contribution, and $\sigma_{i}$ controls its width along the $i$-th direction in the CV space.

Since each replica experiences a different potential due to the bias from the non-Markovian metadynamics terms, as well as to the scaling from the solute tempering scheme, the acceptance probability must be calculated according to the replica exchange prescription with different Hamiltonians [15]. A proposed swap is evaluated according to the Metropolis algorithm, accepting the exchange between the $i$-th and the $j$-th replica with probability given by $P=\min (1, \exp (-\Delta))$, where

$$
\begin{aligned}
\Delta= & \left(\beta_{i}-\beta_{j}\right)\left(E_{p}\left(X_{j}\right)-E_{p}\left(X_{i}\right)\right) \\
+ & \left(\sqrt{\beta_{0} \beta_{i}}-\sqrt{\beta_{0} \beta_{j}}\right)\left(E_{p w}\left(X_{j}\right)-E_{p w}\left(X_{i}\right)\right) \\
- & \beta_{i}\left(V_{i}\left(s\left(X_{i}\right)\right)-V_{i}\left(s\left(X_{j}\right)\right)\right) \\
- & \beta_{j}\left(V_{j}\left(s\left(X_{j}\right)\right)-V_{j}\left(s\left(X_{i}\right)\right)\right) .
\end{aligned}
$$

As already remarked, only the colder replica samples the proper FES, that can be extracted from the limit for large simulation times of the history-dependent potential [10, 11, 12]:

$$
F(\vec{s})=\lim _{t \rightarrow \infty} V_{m}(\vec{s}, t) .
$$

To asses the effect of the metadynamics on the convergence properties of the simulation, we also make a control simulation where we compared the combined approach just described with a run without metadynamics. As shown below in detail, the approach described in this work combines the improved convergence already reported for the parallel tempering metadynamics with a significant reduction in computational effort due to the solute tempering. For example, for a system of $3 \times 10^{4}$ atoms only ten replicas are needed to effectively cover a range of more than $300 \mathrm{~K}$, with respect to more than 60 replicas used for the parallel tempering.

The metadynamics algorithm, solute tempering and combined solute tempering metadynamics have been implemented by the authors on the GROMACS molecular dynamics package [16, 17] and are available upon request. 


\section{B. Simulation details}

The $\alpha$-helix structure studied corresponds to the fragment from the residues 21 through 40 of protein $\mathrm{G}$ (PDB code 1PGB). The interactions were described using the GROMOS 53A6 force field [18, 19], and virtual-site atoms for hydrogens were used to speed up the simulation [20, 21], allowing the time step for the molecular dynamic integration to be as high as $0.004 \mathrm{ps}$. The system was enclosed in a dodecahedron box of $300 \mathrm{~nm}^{3}$ with periodic boundary conditions and solvated with 9856 SPCE water molecules [22]. The system charge was neutralized adding $2 \mathrm{Na}^{+}$ions. Van der Waals interaction were cut-off at $1.4 \mathrm{~nm}$ and the long-range electrostatic interactions were calculated by the particle mesh Ewald algorithm [23] with a mesh spaced $0.125 \mathrm{~nm}$. The neighbor list for the non-bonded interactions was updated every 5 steps (0.020 ps). The system evolves canonically, thermally coupled with a Nosé-Hoover bath [24, 25].

The solvated system was prepared using the following procedure: (1) a steepest descent energy minimization; (2) equilibration of the solvent for 100 ps at $100 \mathrm{~K}$, keeping the heavy atoms of the protein constrained with springs of strength $1000 \mathrm{~kJ} /\left(\mathrm{nm}^{2} \mathrm{~mol}\right)$; followed by another $100 \mathrm{ps}$ at $200 \mathrm{~K}$ with the spring constant reduced to $500 \mathrm{~kJ} /\left(\mathrm{nm}^{2} \mathrm{~mol}\right)$ and by another $100 \mathrm{ps}$ at $300 \mathrm{~K}$ with springs of $250 \mathrm{~kJ} /\left(\mathrm{nm}^{2} \mathrm{~mol}\right)$; (3) a thermal equilibration of the whole system, with a 100 ps dynamics at $300 \mathrm{~K}$, at constant volume; (4) a density equilibration with a 100 ps dynamics at 300K and constant pressure, coupling the system to a Berendsen barostat, and finally (5) a 2 ns dynamics at $300 \mathrm{~K}$ at constant volume to thermalize again the system.

After such preparation, we run 10 replicas of the system (respectively at 300, 325, 352, 381, 413, 447, 484, 524, 568 and $615 \mathrm{~K}$ ), attempting to swap neighbors replicas every 50 steps, $\tau_{x}=0.2 \mathrm{ps}$. We verify a posteriori that choosing these temperatures we achieve an optimal acceptance rate (between $30 \%$ and $40 \%$ ).

Regarding the acceptance rate of the proposed swaps between replicas, we observed a significant increase from the solute tempering metadynamics (where it was about 35\%) and the simple solute tempering case, where the same temperature choices reduced the rate to $25 \%$.

Moreover in ref. [26] Huang, et al. shows that solute tempering is not efficient for proteins because not taking into account the water-water interaction energy in the replica exchange 
acceptance probability reduce greatly the exchanges between folded and unfolded conformations. In the particular case under study we have seen that each replica explores the whole temperature range or for solute tempering either for solute tempering metadynamics. However, for the last approach an increase of exchange probability was observed.

The choice of the collective variables included in the metadynamics reflects the physical insight of the problem being addressed. In order to describe the folding of the helix, we therefore choose as collective variables the radius of gyration of the backbone heavy atoms and the number hydrogen bonds between backbone atoms of residues 4 sites apart along the sequence $(i, i+4$ hydrogen bonds). Since the algorithm requires the collective variables to be differentiable functions of the microscopic coordinates, we evaluated this count as

$$
N_{H}=\sum_{i=2}^{13} \frac{1-\left(r_{i, i+4} / d_{0}\right)^{n}}{1-\left(r_{i, i+4} / d_{0}\right)^{m}},
$$

with $n=6$ and $m=10, d_{0}=0.32 \mathrm{~nm}$, and where $r_{i, i+4}$ is the distance between the backbone oxygen of residue $i$ and the backbone nitrogen of residue $i+4$.

Clearly, there is a trade-off in the choice of the values of $m$ and $n$. A better discrimination between formed and non-formed H-bonds entails that an higher number of conformations display a vanishing value of the gradient $\partial N_{H} / \partial r_{i, i+4}$. For these conformations, the historydependent potential has little effect on the dynamics, and thus the algorithm looses its effectiveness in correctly sampling all the relevant phase-space.

Moreover, it is important to notice that the functional form that defines this CV does not contain any angular contribution; thus, conformations in which one oxygen at site $i$ falls close to one nitrogen at site $i+4$ will contribute to the CV even if their positions do not satisfy the angular criterion determining an $\mathrm{H}$-bond (i.e. $\theta_{\mathrm{H}-\mathrm{N}-\mathrm{O}}<30^{\circ}$ ). Again, including such criterion would on one hand improve the accuracy with which the CV describes the formation of helices, but on the other hand, enlarge the region of the phase space in which the gradient of the $\mathrm{CV}$ with respect to the microscopic atomic coordinates vanishes. The chosen values of the exponents $m$ and $n$ guarantee that the derivatives along the range of distances $r_{i, i+4}$ never vanish to the numerical precision. This comes with the price of a reduced accuracy in identifying the exact number of formed $i-(i+4) \mathrm{H}$-bonds.

Notice that these variables do not require any specific knowledge of the folded structure.

We choose a Gaussian height $w_{m}=0.7 k_{B} T_{m}$, a width $\sigma_{\mathrm{Gyr}}$ of $0.01 \mathrm{~nm}$ and $\sigma_{\mathrm{Hb}}$ of $0.1 \mathrm{~nm}$. The bias potential is updated every 125 steps, $\tau_{G}=0.5 \mathrm{ps}$. 


\section{RESULTS AND DISCUSSION}

The free energy of the helix as a function of the CV defined above is shown in Fig. 1 for different durations of the simulations. The free-energy is depicted every $0.7 \mathrm{~ns}$ in order to inspect the convergence of the surface along the metadynamics simulation. The theoretical boundaries for the $\mathrm{CV}$ are from 0 to 8 for the hydrogen-bond number and from $0.6 \mathrm{~nm}$ to $1.8 \mathrm{~nm}$ for the gyration radius, corresponding to fully compact and fully extended conformations, respectively. From these calculations we have found that it takes about 6 ns for metadynamics to explore the whole (accessible range) of the phase space. No change in the structure of the minimum and of the transition states takes place afterward, suggesting that the free energy has converged to its equilibrium shape.

As a further check of equilibration we performed another simulation, applying solute tempering metadynamics to a system which, instead of starting from the folded conformation as in the former case, started from an extended conformation. The resulting FES converged (to the same accuracy) to the previously calculated FES, within the same time span (data not shown).

The most relevant meta-stable structures obtained form the simulations are reported in Fig. 2, along with their position on the FES landscape. In the unfolded region, we observe several minima, each of which characterize a different representative structures of the unfolded state. Referring to the labels indicated in Fig. 2, state (a) corresponds to a molten globule conformation, featuring a very small gyration radius. This state is separated from the remainder of the phase space by a high barrier (about $20 \mathrm{~kJ} / \mathrm{mol}$ ). State (b) corresponds to a $\beta$-bridged structure, with a well defined turn spanning the residues 28 to 30. Another extended state, corresponding to a random coil conformation is located at an even higher gyration radius, and is labeled as state (c). Finally, state (d), displays one $\alpha$-turn near the $\mathrm{N}$-terminus region.

As far as the folded region is concerned we find two different conformations in the corresponding FES landscape ((e) and (f), Fig. 2), they correspond to an $\alpha$-helix (featuring hydrogen bonds between residues $i$ and $i+4$ ) and a $\pi$-helix (bonds between residues $i$ and $i+5$ ), respectively. Interestingly, the folded helix in the protein (see (g), Fig. 2) displays the last loop in a $i-(i+5)$ conformation. The two structures displayed in Fig. 2 (e) and (f) are not discriminated by the chosen CV since the contribution of the distance between 
residues $i, i+4$ to the $N_{H}$ collective variable in the $\pi$-helix is still large. Consequently, we cannot assess, even qualitatively, the relative stability of the two structures.

Finally, notice that the structure of the helix in the protein, indicated in Fig. 2 as (g), lies at a even higher value of the hydrogen-bond collective variable than do the conformations (e) and (f) (Fig. 2), and does not correspond to a minimum.

In [13] it was shown that the combination of parallel tempering and metadynamics shows a significant improvement in convergence with respect to the simple parallel tempering case. We thus expect that a similar synergy be present also in the case under discussion, since solute tempering is expected to behave much like parallel tempering. To check that this is indeed the case, we performed a solute tempering simulation for $8 \mathrm{~ns}$ on the system, monitoring the behavior of the two CV every 125 steps, (0.5 ps). A FES was extracted from such run and is shown in Fig. 3.

As far as the solute tempering simulation is concerned, it is clear from Fig. 3 that neither the folded nor the unfolded region is converged even after $8 \mathrm{~ns}$, and that the area of the phase space explored is significantly smaller than in the case reported in Fig. 2, Another indication of the broader exploration of the configuration space carried out by solute tempering metadynamics compared to simple solute tempering, is given by the number of clusters explored. Clustering the structures in the trajectory with a linkage method (using a cutoff of $0.15 \mathrm{~nm}$, and considering the backbone RMSD), we obtain 163 clusters for the first $6.3 \mathrm{~ns}$ of the solute tempering trajectory, and 398 clusters for the solute tempering metadynamics. Even extending the solute tempering to 8 ns, only 248 clusters are sampled.

Experimental investigations carried out either through nuclear magnetic resonance or circular dichroism [4, 5] have been done on a peptide identical to the one studied in this work but for the $\mathrm{V} 21 \mathrm{G}$ mutation. The helical structure is found not to be stable in water; in particular CD spectroscopy allows to estimate a possible helical content of only $5 \%$ at 270 $\mathrm{K}$. This value is in qualitative agreement with the estimate which can be done with the help of a two-state picture of the calculated free-energy at $300 \mathrm{~K}$. In fact, we obtain a free-energy difference between the folded and the unfolded states of $\Delta G_{F U} \approx 9 \mathrm{~kJ} / \mathrm{mol}$, giving $2 \%$ for the probability of being in an helical state. Furthermore, the same experimental work indicate that the helical region in water comprises the residues from D22 to Q32 in accordance with the observed structures in (e), at variance with the longer range in the protein structure $(\mathrm{g})$ that extends up to N37. We notice that as far as the $\pi$-helix (f) is concerned, our results 
show that these structures are helical up to N37 even in water.

Another feature of the calculated FES which can be compared with experimental evidence is the presence of partial $\beta$-structures. Nuclear magnetic resonance data [4] shows that residues K28 to K31 are significantly populating the $\beta$-region of the $(\phi, \psi)$ space. This is consistent with the finding of very stable conformations within such region of the Ramachandran plot in configurations (b).

An interesting point concerning the stability of the helix regards the presence of the socalled hydrophobic staple motif, Fig. 4. Such motif is not observed in the protein structure, despite the favorable sequence at the N-terminus of the helix. Blanco et al. [5] however, report nuclear magnetic resonance results which indicate the likely presence of such stabilizing motif in the isolated helix in water.

Analyzing the most representative structures in the folded conformations (structures $(e)$ and $(f)$ in Fig. 2), we checked that the side-chain of V21 and A26 are in contact, as well as those of D22 and T25, indicating that indeed the staple motif stabilized both the $\alpha$ - and the $\pi$ - helical structures.

A further analysis which is usually done starting from FES consists in the identification of the most probable kinetic trajectory identified with the minimum pathway between pairs of thermodynamic states [27, 28]. For this purpose, a careful choice of the CV is critical [27]. On the contrary, the use of the solute tempering coupled with metadynamics used in the present work is more tolerant to a loose choice of the CV. An example of this issue is provided by the states labeled (e) and (f) in Fig. 2. This is a single minimum in the free energy calculated as a function of $N_{H}$ and $R_{g}$, but it contains two sets of conformations, that is $\alpha$ - and $\pi$-helices, which are certainly separated by energy barriers. In other words, $\alpha$ - and $\pi$-helices would require, to be distinguished in solute tempering metadynamics simulation, more (or different) CV. From a kinetic point of view, this is a serious obstacle for a correct description of the folding trajectory. On the other hand, the use of these CV allows solute tempering metadynamics for an efficient reconstruction of the free energy landscape.

What can be done instead is to describe the formation of the helix only from a qualitative point of view, analyzing the features of a representative set of conformations extracted from the local-minimum basin (i.e. the extended folded region around the points (e) and (f) in Fig. 2). We define operatively the basin as the set of conformations displaying a number of H-bonds between 3 and 5 and a gyration radius between $0.70 \mathrm{~nm}$ and $0.72 \mathrm{~nm}$. One can 
observe that the structures with fewer H-bonds comprise two-formed loops in the region from 25 to 33, i.e. in the N-terminus half of the chain. No structures with formed loops in the C-terminus are observed. One can thus infer that, once these loops are formed, the helix grows on both sides, reaching (e) and (f). This is consistent with the fact that the primary sequence of the peptide displays a larger helical propensity in the N-terminus region than in

the C-terminus region [5]. This result seems to point against the suggestion reported in [7], where Baker and coworkers suggest, on the basis of side-directed mutagenesis studies on the entire protein, that the helix grows from the C-terminal. As far as the staple motif is concerned, an analysis of the same conformations shows that the contact between V21 and A26 and the hydrogen bond between D22 and T25 are formed only for structures where the helix is folded completely, i.e. in (e) and (f). No presence of the staple motif is observed when the helix displays only part of the turns formed.

\section{CONCLUSIONS}

We have calculated and analyzed the FES of a fragment of Protein G corresponding to a helical structure in the protein, efficiently sampling the conformations of the system using a combined solute tempering and metadynamics algorithm.

We show that the proposed algorithm is able to explore a broad region of the phase space and allows to accurately estimate the associated free energy within affordable computational effort. Several experimental findings can be reproduced and explained with help of the simulation. In particular, we find that the helix is not stable in water, and that the metastable $\alpha$-helical state is shorter than the helix found in the native state of the protein. We also observe the presence of partial $\beta$-structures in the unfolded state. Furthermore, the simulations indicates that the peptide populates not only an $\alpha$-helical conformation, but also a $\pi$-helix, a result which can be tested experimentally.

As far as the dynamics of helix formation is concerned, the simulation indicate that both kinds of helices grow from the N-terminus half. The role of non-native interactions in stabilizing the helical fold is also confirmed by the presence of an hydrophobic staple motif, also located at the N-terminus of the helix.

Solute tempering metadynamics shows remarkable robustness against a partial knowledge of all the relevant slow degrees-of-freedom characterizing the systems, guaranteeing an 
exhaustive free-energy sampling in spite of the use of degenerate collective variables (which, for example, are not able to distinguish between $\alpha$ - and $\pi$-helices).

Summing up, the thermodynamics of two of the most important secondary structures of protein $\mathrm{G}$ have been characterized by two different flavors of replica exchange metadynamics methods (the $\alpha$-helix by us and the second $\beta$ - hairpin by Bussi, at al. [13]). The efficiency of the solute tempering method described here and its speed, makes it particularly suited to attack larger systems, in particular the whole of protein G. 
[1] Gronenborn AM, Filpula DR, Essig NZ, Achari A, Whitlow M, Wingfield PT, Clore GM. A novel, highly stable fold of the immunoglobulin binding domain of streptococcal protein $G$. Science 1991; 253:657-661.

[2] Alexander P, Orban J, Bryan P. Kinetic Analysis of Folding and Unfolding the 56 Amino Acid IgG-Binding Domain of Streptococcal Protein G. Biochem. 1992; 31:7243-7248.

[3] Alexander P, Fahnestock S, Lee T, Orban J, Bryan P. Thermodynamic Analysis of the Folding of the Streptococcal Protein G IgG-Binding Domains B1 and B2: Why Small Proteins Tend To Have High Denaturation Temperatures. Biochem. 1992; 31:3597-3603.

[4] Blanco FJ, Serrano L. Folding of protein G B1 domain studied by the conformation characterization of fragments comprising its secondary structure elements. Eur. J. Biochem. 1995; 230:634-649.

[5] Blanco FJ, Ortiz AR, Serrano L. Role of nonnative interaction in the folding of the protein $G B 1$ domain as inferred from the conformational analysis of the $\alpha$-helix fragment. Fold. Des. $1997 ; 2: 123-133$.

[6] Cregut D, Civera C, Macias MJ, Wallon G, Serrano L. A tale of two secondary structure elements: when a $\beta$-hairpin becomes an $\alpha$-helix. J. Mol. Biol. 1999; 292:389-401.

[7] McCallister EL, Alm E, Baker D. Critical role of $\beta$-hairpin formation in protein $G$ folding. Nat. Struct. Biol. 2000; 7:669-673.

[8] Hansmann UHE. Parallel Tempering Algorithm for Conformational Studies of Biological Molecules. Chem. Phys. Lett. 1997; 281:140-150.

[9] Sugita Y, Okamoto Y. Replica-exchange molecular dynamics method for protein folding. Chem. Phys. Lett. 1999; 314:141-151.

[10] Laio A, Parrinello M. Escaping free-energy minima. Proc. Natl. Acad. Sci. U.S.A. 2002; 99:12562-12566.

[11] Laio A, Rodriguez-Fortea A, Gervasio FL, Ceccarelli M, Parrinello M. Assessing the accuracy of metadynamics. J. Phys. Chem. B 2005; 109:6714-6721.

[12] Bussi G, Laio A, Parrinello M. Equilibrium free energies from nonequilibrium metadynamics. Phys. Rev. Lett. 2006; 96:090601.

[13] Bussi G, Gervasio FL, Laio A, Parrinello M. Free-Energy Landscape for $\beta$ Hairpin Folding from 
Combined Parallel Tempering and Metadynamics. J. Am. Chem. Soc. 2006; 128:13435-13441.

[14] Liu P, Kim B, Friesner RA, Berne BJ. Replica exchange with solute tempering: A method for sampling biological systems in explicit water. Proc. Natl. Acad. Sci. U.S.A. 2005; 102:1374913654.

[15] Yan Q, de Pablo JJ. Hyper-parallel tempering Monte Carlo: Application to the Lennard-Jones fluid and the restricted primitive model. J. Chem. Phys. 1999; 111:9509-9516.

[16] Berendsen HJC, van der Spoel D, van Drunen R. GROMACS: A message-passing parallel molecular dynamics implementation. Comp. Phys. Comm. 1995; 91:43-56.

[17] Lindahl E, Hess B, van der Spoel D. GROMACS 3.0: A package for molecular simulation and trajectory analysis. J. Mol. Mod. 2001; 7:306-317.

[18] Oostenbrink C, Villa A, Mark AE, Van Gunsteren WF. A biomolecular force field based on the free enthalpy of hydration and solvation: The GROMOS force-field parameter sets 53 A5 and 53A6. J. Comp. Chem. 2004; 25:1656-1676.

[19] Oostenbrink C, Soares TA, van der Vegt NFA, van Gunsteren WF. Validation of the 53A6 GROMOS force field. Eur. Biophys. J. 2005; 34:273-284.

[20] Miyamoto S, Kollman PA. SETTLE: An Analytical Version of the SHAKE and RATTLE Algorithms for Rigid Water Models. J. Comp. Chem. 1992; 13:952-962.

[21] Hess B, Bekker H, Berendsen HJC, Fraaije JGEM. LINCS: A Linear Constraint Solver for molecular simulations. J. Comp. Chem. 1997; 18:1463-1472.

[22] Berendsen HJC, Grigera JR, Straatsma TP. The Missing Term in Effective Pair Potentials. J. Phys. Chem. 1987; 91:6269-6271.

[23] Essman U, Perela L, Berkowitz ML, Darden T, Lee H, Pedersen LG. A smooth particle mesh Ewald method. J. Chem. Phys. 1995; 103:8577-8592.

[24] Nosé S. A molecular dynamics method for simulations in the canonical ensemble. Mol. Phys. $1984 ; 52: 255-268$.

[25] Hoover WG. Canonical dynamics: equilibrium phase-space distributions. Phys. Rev. A 1985; 31:1695-1697.

[26] Huang X, Hagen M, Kim B, Friesner RA, Zhou R, Berne BJ. Replica exchange with solute tempering: efficiency in large scale systems. J. Phys. Chem. B 2007; 111:5405-5410.

[27] Bolhuis PG, Chandler D, Dellago C, Geissler PL. Transition path sampling: throwing ropes over rough mountain passes, in the dark. Annu. Rev. Phys. Chem. 2002; 53:291-318. 
[28] Bonomi M, Gervasio FL, Tiana G, Provasi D, Broglia RA, Parrinello M. Mechanistic insight in the folding inhibition of the HIV-1 Protease by a small peptide. Biophys. J. 2007; (in press). 

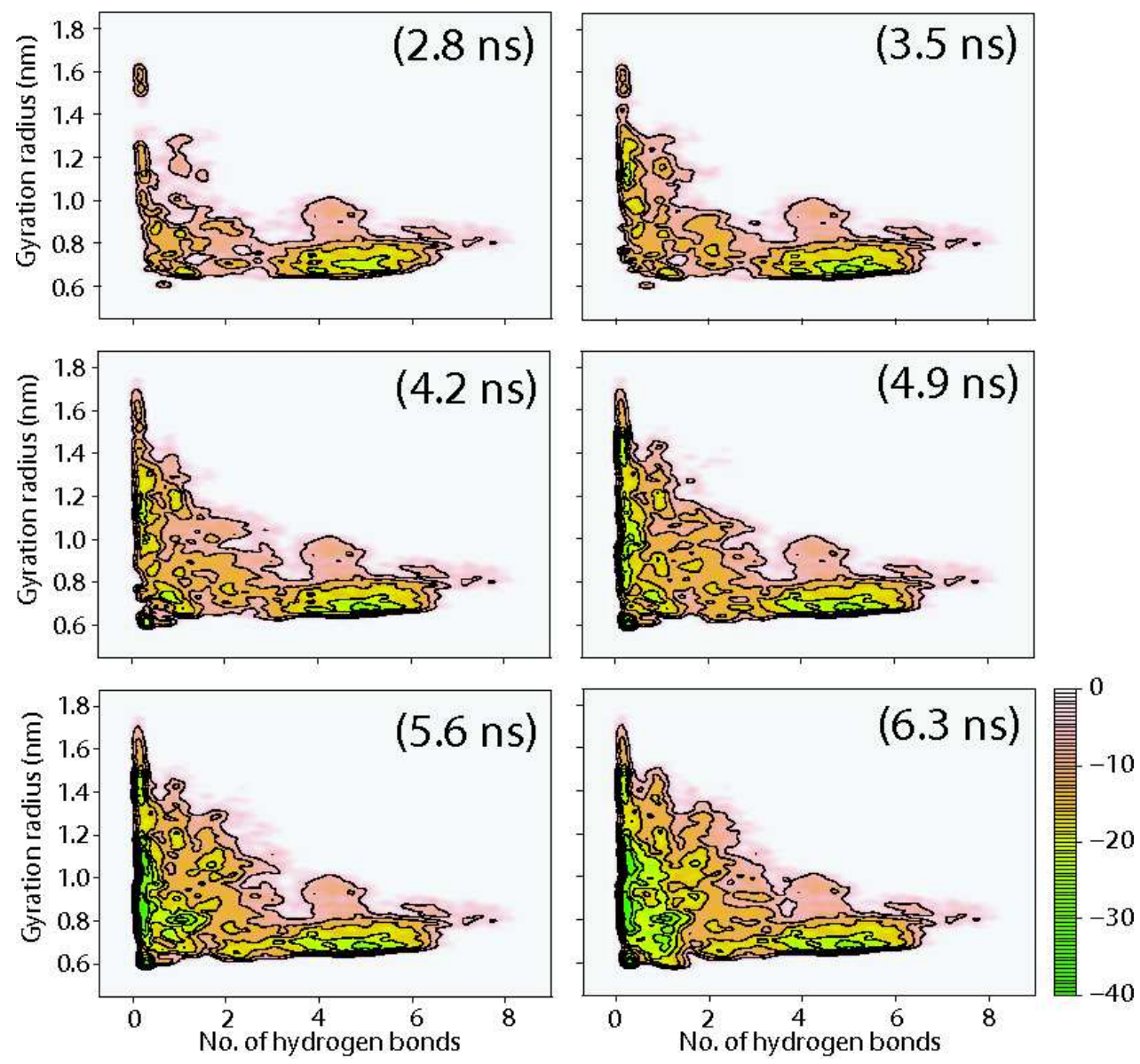

FIG. 1: Evolution and convergence. Free-energy surface of the helix. The calculated FES are reported every 0.7 ns until convergence is achieved after about 6.3 ns. Free-energy is reported in $\mathrm{kJ} / \mathrm{mol}$ and contour lines are $5 \mathrm{~kJ} / \mathrm{mol}$ apart. 


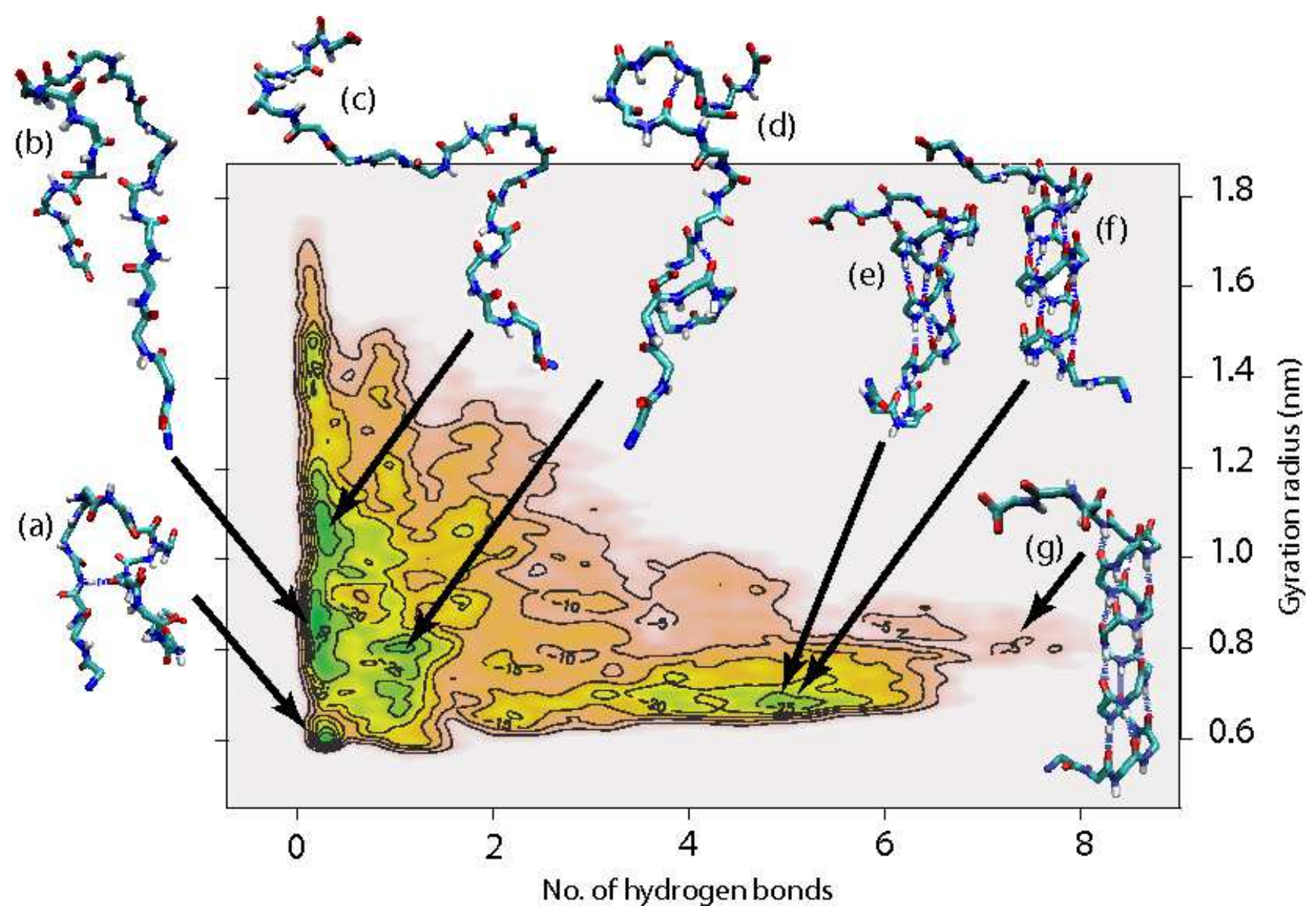

FIG. 2: Relevant meta- and stable structures. Converged FES of the helix (after 6.3 ns of metadynamics). The most representative structures of each local minimum have been represented. Free-energy is reported in $\mathrm{kJ} / \mathrm{mol}$ and contour lines are $5 \mathrm{~kJ} / \mathrm{mol}$ apart. 


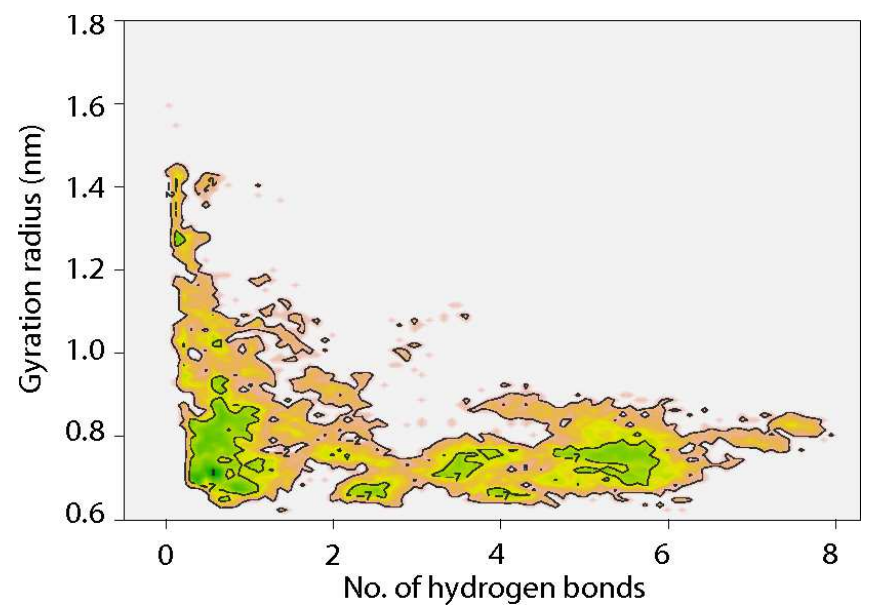

FIG. 3: Solute tempering simulation. Free-energy surface of the helix extracted from a $8 \mathrm{~ns}$ solute tempering simulation. Despite the longer sampling, a comparison with the solute tempering metadynamics results (cfr. Fig. 22) shows that not all the relevant configurations have been reached.

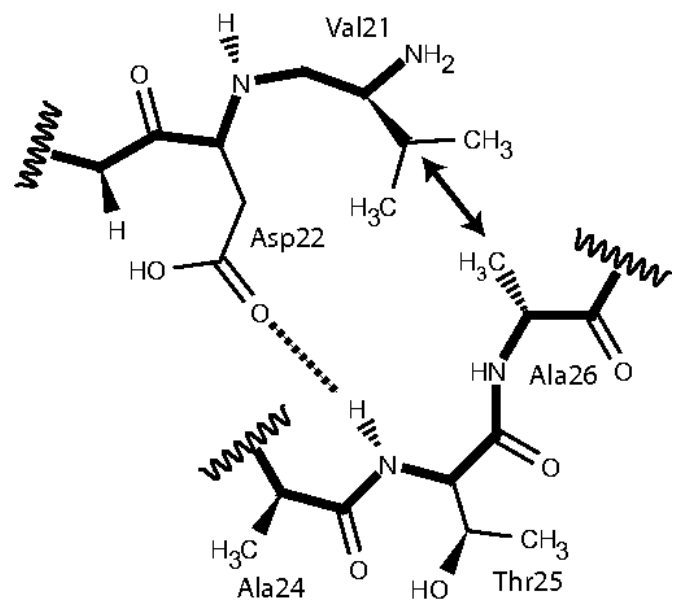

FIG. 4: Hydrophobic staple motif. Schematic representation of the hydrophobic staple motif observed in the folded conformations (structures (e) and (f) in Fig. 3). The observed contact between V21 and A26 is indicated by a two-way arrow, while the observed hydrogen bond between D22 and T25 with a dotted line. 